Alain SOMAT \& Pascal Morchain

\title{
Introduction
}

\section{DE LA VITALITÉ DE LA RECHERCHE EN PSYCHOLOGIE SOCIALE APPLIQUÉE}

\section{De L'UTILITÉ de LA PUblication D'Un OUVRAGE DE PSYCHOLOGIE SOCIALE APPLIQUÉE}

$35000^{1}$. Ce serait le nombre de psychologues actuellement en activité en France. Environ 45 \% d'entre eux déploient leur talent dans la fonction publique d'état ou territoriale, $30 \%$ travaillent dans le secteur associatif, $10 \%$ sont en libéral et 5 à $10 \%$ exercent dans le secteur privé. Ces psychologues sont formés par l'université à bac plus 5 dans le cadre d'une licence de psychologie puis d'un master de psychologie. Fort de leur diplôme acquis à l'université, ils peuvent s'engager dans une inscription auprès des DDASS ou des DRASS sur une liste dite « ADELI » (Automatisation DEs LIstes). Il s'agit d'un système d'information national sur les professionnels relevant du Code de la santé publique, du Code de l'action sociale et des personnes autorisées à faire usage du titre de psychologue. Il contient des informations comme l'état civil, la situation professionnelle, les activités exercées. Il permet de gérer les listes départementales de professions réglementées par le Code de la santé publique, le Code de l'action sociale et des familles et celles des personnes autorisées à faire usage du titre de psychologue. Il permet également d'élaborer des statistiques permettant une meilleure planification de l'évolution démographique des professions et d'informer les professionnels. Un numéro ADELI est attribué à tous les praticiens salariés ou libéraux et leur sert de numéro de référence. Le numéro ADELI figure sur la carte de professionnel de santé (CPS) pour des professionnels relevant du Code

1. Rosenzweig $(1992,1999)$ affirme que les psychologues sont plus de 500000 au niveau mondial. 
de la santé publique. Bien que disposant par convention collective d'un temps DIRES (Documentation, Information, Recherche, Élaboration, Supervision) ou par circulaire (DH/FH3/92 n 23 du 23 juin 1992, relative à l'application du décret du 31 janvier 1991) d'un temps FIR (Formations Informations Recherches) les psychologues n'ont aucune obligation, sinon morale, de formation continue pour conserver leur titre de psychologue ${ }^{2}$. Mais ce temps de formation légale ou par engagement verbal de l'employeur (temps FIR ou DIRES) conduit les psychologues à conserver tout au long de leur carrière un certain contact avec l'évolution de la discipline. Certains d'entre eux délivrent des enseignements au sein de nos départements ou UFR de psychologie et gardent ainsi contact avec leurs enseignants et le développement de leur champ d'exercice. D'autres lisent les récents développements des travaux de leur sousdiscipline d'appartenance. Ils disposent pour cela d'excellents supports comme Pratiques Psychologiques ou Psychologie Française. Quelques-uns gardent également le contact par leur appartenance à de grandes organisations de psychologues comme la SFP (Société française de psychologie) ou la FFPP (Fédération française des psychologues et de psychologie). D'autres encore, alors qu'ils sont confrontés à une difficulté professionnelle, adhèrent à un syndicat national spécifique à la profession comme le SNP (Syndicat national des psychologues). Bref, d'une manière ou d'une autre les psychologues savent (et cela leur a été très largement inculqué durant leur cursus) que la discipline est en constante évolution. Que la psychologie d'hier n'est pas celle d'aujourd'hui, qui elle-même n'a rien à voir avec celle de demain. Ainsi, conscients de cette rapide évolution signe du développement de la discipline, les psychologues ont volonté et possibilité de maintenir, de confirmer leur savoir et même de

2. On peut penser qu'à court terme une certification européenne conduira les psychologues, pour obtenir la certification EUROPSY (certification de l'European Federation of Psychologist Association), à faire valoir une preuve de la formation continue réalisé au cours d'une période de 5 ans. De fait, le 17 janvier 2009 s'est tenue à Oslo une réunion européenne sur la mise en œuvre d'EuroPsy dans les différents pays membres de l'EFPA. À cette réunion assistaient le comité exécutif de l'EFPA, le groupe de pilotage d'EuroPsy, et les représentants de 30 des 34 organisations nationales membres de l'EFPA. Le 30 janvier s'est réuni pour la première fois à Paris le CoFraDeC EuroPsy. Cette première réunion a été consacrée pour l'essentiel à une prise de contact et à une discussion générale sur le projet EuroPsy, et les conditions de son application en France. Chacun des membres du comité a pu expliciter sa manière de concevoir la mise en œuvre d'un tel projet. Il s'est dégagé un consensus très clair sur les principes de base qui doivent guider l'action du comité qui a organisé le 21 novembre 2009 sa première publique pour élaborer avec l'ensemble de la profession les bases de cette mise en place. 
le développer en vue de demeurer au niveau de compétences que leur a octroyé leur diplôme délivré à bac plus 5 . Le temps dont disposent les praticiens et la pratique de leur activité les portent vers la recherche d'applications des savoirs fondamentaux issus de nos laboratoires. Or, reconnaissons que peu d'ouvrages se donnent cette ambition pourtant fondamentale de mettre à disposition des professionnels concernés un état des lieux régulier en terme d'application d'un champ de recherches universitaires. Or, si l'on veut que nos collègues praticiens disposent des récentes avancées de nos travaux, il nous faut considérer, bien que cet exercice soit peu rentable au regard du facteur d'impact, que cet exercice relève de notre activité. Garder cet objectif est fondamental si l'on souhaite que la psychologie conserve le niveau d'exigence que la profession lui accorde : un niveau de formation équivalent à celui que l'on délivre à un ingénieur. Cet ouvrage participe d'un tel projet et fournit une présentation du développement d'une discipline (la psychologie sociale) qui presque depuis ses origines s'interroge sur son utilité pour les différents champs professionnels (santé, travail, éducation et formation) dans lesquels on la convoque ou vers lesquels elle se porte.

\section{QUELQUES DONNÉES DE L'HISTORIQUE DES APPLICATIONS EN PSYCHOLOGIE}

De fait, depuis 1920 l'Association internationale des conférences de psychotechniques puis l'IAAP (International Association of Applied Psychology) dont le premier congrès a eu lieu en 1920 à Genève sous la présidence d'Édouard Claparède servait cet objectif du développement d'une recherche appliquée en psychologie. Souvenons-nous, ce premier congrès réunissait seulement 17 psychologues représentant déjà 8 nations ${ }^{3}$ et a été à l'origine d'une longue série que l'on peut à la suite de Wilpert (1998) puis de Fleishman (1979, 1999) catégoriser en 4 époques. En premier lieu, l'époque de la mise en place de 1920 à 1930 durant laquelle la psychologie appliquée se limite à une approche psychotechnique. La principale préoccupation des applications en psychologie se limite alors à la production d'outils au service de l'organisation du travail et de l'évaluation scolaire. Période agitée de l'histoire du xx siècle, les années 1931 à 1937 verront les psychologues s'emparer des problèmes sociaux de l'époque en développant des recherches appliquées dans les domaines de la sociopolitique et de la solidarité entre les

3. Dont la France, d'ailleurs : le $4^{\mathrm{e}}$ congrès sera organisé à Paris et réunira 341 participants. 
personnes. Suspendues de 1938 à 1949, les activités de l'IAAP reprendront de manière active dès 1949 avec le congrès de Berne (Suisse) sous la présidence d'Henri Piéron pour se déployer jusqu'en 1955 dans le cadre d'applications portant principalement sur la résolution de conflit dans de nombreux et divers champs sociaux. De 1964 à nos jours, l'association internationale trouve sa vitesse de croisière par l'organisation tous les 4 ans d'un congrès international qui rassemble entre 3000 et 5000 participants. Les centres d'intérêt sont très variés et s'organisent autour des 14 divisions de l'IAAP. Ces champs d'applications couvrent, nous semblet-il, l'ensemble des secteurs d'activité de la psychologie appliquée. Ainsi, les psychologues praticiens peuvent, de fait, y trouver leur intérêt. Pour preuve, la répartition se fait en secteurs d'activité de la manière suivante :

1. Division 1 : Psychologie du travail et des organisations.

2. Division 2 : Évaluation des processus psychologiques.

3. Division 4 : Psychologie environnementale.

4. Division 5 : Psychologie scolaire, éducation et processus éducatif.

5. Division 6 : Clinique et psychologie communautaire.

6. Division 7 : Psychologie gérontologique.

7. Division 8 : Psychologie de la santé.

8. Division 9 : Psychologie économique.

9. Division 10 : Psychologie et justice.

10. Division 11 : Psychologie politique.

11. Division 12 : Psychologie du sport.

12. Division 13 : Psychologie des transports et de la sécurité routière.

13. Division 14 : Psychologie cognitive appliquée.

14. Division 16 : Psychologie conseil et relations d'aide.

À ces 14 sections relatives à des champs d'application, il faut rajouter les 3 secteurs sur lesquels l'IAAP souhaite garder une certaine vigilance : les pratiques professionnelles (division 3), le développement de la psychologie (division 15), et la formation en psychologie et l'orientation professionnelle des psychologues (division 17).

Les congrès de l'IAAP, dont plusieurs se sont déroulés en Europe (Barcelone : 1921, 1930, Milan : 1922, Paris : 1927, 1953, Utrecht : 1928, Prague : 1934), constituent l'histoire des applications en psychologie. Histoire à laquelle la France va une nouvelle fois contribuer en 2014 lorsque Paris, sous la présidence du professeur Roland Lévy, organisera le $28^{e}$ congrès de l'IAAP. Ce congrès devrait constituer pour la communauté des 35000 psychologues français une formidable opportunité pour faire en sorte que la psychologie française se donne les moyens de faire la démons- 
tration de son extraordinaire potentiel de recherche applicable voire appliquée. Ce sera aussi une occasion unique, qu'il ne faudra pas rater, de parfaire nos connaissances dans divers domaines d'application et peut être développé des réseaux de recherches et de collaboration internationale.

On le voit la recherche appliquée en psychologie possède en France une réelle vitalité. Elle a, nous en sommes tous convaincus, son utilité, et elle est l'objet d'un véritable dynamisme au niveau national comme international. Pourtant assez peu d'ouvrages sont publiés chaque année pour mettre à disposition du plus large public les avancées significatives sur les applications.

\section{QUELQUES ARGUMENTS POUR UN NOUVEL OUVRAGE EN PSYCHOLOGIE SOCIALE APPLIQUÉE}

Le projet de cet ouvrage est né à la suite d'un congrès de l'ADRIPS (Association pour la diffusion de la recherche internationale en psychologie sociale, 2005) au cours duquel nous avions souhaité organiser une manifestation restreinte de manière à disposer du temps nécessaire à la réflexion, à la discussion et aux échanges sur ce que sont les applications d'une sous-discipline de la psychologie. Comme il s'agissait d'une communauté de psychologues sociaux, les exposés et les débats se sont déployés dans le cadre de travaux issus de près ou de loin des champs d'activité classique de la psychologie sociale. Chacun s'était donné pour objectif de livrer à la communauté présente un état de la question sur son domaine de spécialité dans le but de s'interroger de l'applicabilité de son champ de recherche et des éventuelles applications réalisées durant ces dernières années. Cette façon de voir les choses a conduit les communicants à échanger sur la signification de la volonté d'appliquer un champ de recherche fondamentale. D'une manière ou d'une autre, les propos ont été une illustration de l'idée à laquelle nous tenons au sein de notre laboratoire (LAUREPS) sur ce qu'est l'ingénierie en psychologie, et plus particulièrement l'ingénierie en psychologie sociale. D'une manière ou d'une autre les arguments déployés l'ont été pour « convaincre de l'utilité de la psychologie sociale dans différents secteurs en montrant que ses apports ne se limitent pas à octroyer un supplément d'âme ou un éventuel " plus » culturel à des professionnels, mais peuvent aider efficacement à la compréhension de phénomènes psychosociaux et à la conception de dispositifs impliquant des personnes, des groupes ou des organisations » (Guingouain \& Le Poultier, 1994, p. 4). Toutes les communications ont participé de l'idée que la discipline possède les 
moyens de produire un savoir qui confère aux psychologues sociaux des « utilités d'ingénieur» (Beauvois \& Ghiglione, 1989). Ces exposés (brillants au demeurant) ont été une illustration de l'idée selon laquelle s'engager dans une démarche d'application renvoie à la satisfaction de trois obligations incontournables. (1) Les applications en psychologie sociale doivent avoir un arrière-plan théorique et méthodologique solide construit à l'aide d'expérimentations. (2) Appliquer nécessite une analyse serrée et complexe du contexte d'insertion sociale de l'événement ou de la situation étudiés. (3) Appliquer suppose une compétence à concevoir et à mettre en œuvre des propositions concrètes de modification du système étudié, et à appréhender les conséquences de cette modification. Py, Baillé et Somat (1998), Py et Somat (2007), Py et Somat (à venir) et Pansu, Py et Somat (à venir) proposent un modèle dit d'ingénierie psychosociale pour s'engager dans une telle démarche de recherche appliquée. Cette proposition tient dans l'articulation entre différents types de recherches : étude d'archives, recherche évaluative, recherche fondamentale, recherche appliquée de laboratoire, recherche expérimentale de terrain, recherche-action (voir schéma ci-dessous).

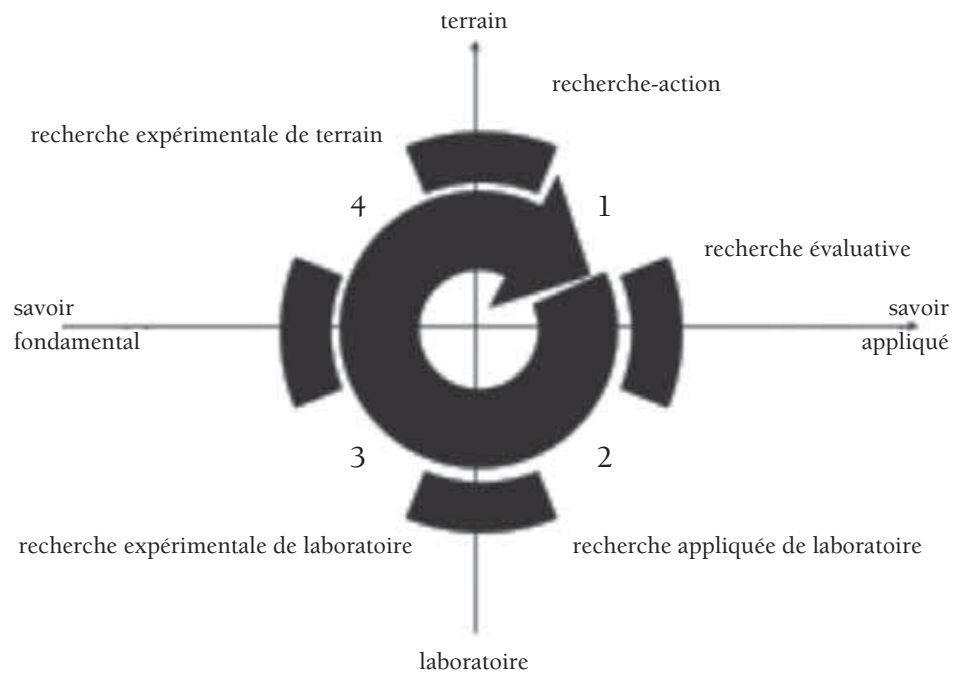

Cette démarche procède de la mise en œuvre des 4 étapes suivantes : $1^{\mathrm{e}}$ étape : Évaluer un problème de terrain (audit) en vue de déterminer le problème de terrain à traiter, en tentant d'évaluer l'efficacité des procédures existantes et les biais et insuffisances. Dans le cadre de cette activité d'évaluation d'une problématique, Jacques, Somat, Aldrovandi, 
Guingouain, et Rault (2002) ont décrit les mécanismes d'intériorisation des valeurs d'une entreprise à l'œuvre dans une organisation venant tout juste de mettre en place un style de management participatif.

$2^{\mathrm{e}}$ étape : Conceptualiser une solution alternative : Il s'agira d'envisager un ensemble de pistes possibles permettant de dépasser le constat établi lors du bilan en tenant compte à la fois (a) des biais et insuffisances relevées lors des observations et (b) des apports disponibles dans la littérature scientifique. Si ces apports s'avèrent insuffisants, il conviendra de construire les savoirs nécessaires. Kergreis, Somat et Testé (2003, 2009) ont ainsi travaillé sur la question des bordures de champs (haies et bandes herbeuses) faisant l'objet de contestations sociales et de politiques publiques en Bretagne.

$3^{e}$ étape : Construire un modèle d'action par la comparaison de l'efficacité des différentes propositions alternatives et des pratiques de terrain habituelles. Matuchet, Somat, Testé et Lucet (2005) ont construit et mis en place un outil d'évaluation $360^{\circ}$ dont les fondements trouvent leur origine dans les célèbres travaux de Moreno sur la sociométrie.

$4^{\mathrm{e}}$ phase : Appliquer le modèle d'action par la mise au point de formations professionnelles pour diffuser le modèle d'action validé puis par l'évaluation de ces formations et enfin par un accompagnement du changement des pratiques professionnelles modifiées. Férec, Pansu, Py, et Somat (en révision) se sont par exemple intéressés à la formation à l'internalité et à la clairvoyance normative chez des chômeurs de longue durée.

Pour donner une illustration de l'ensemble de la démarche, voyons les travaux de Demarchi et Py (2006) sur l'entretien cognitif. Le champ de recherche appliquée sur le recueil des témoignages (qu'il s'agisse de témoignages relatifs à des crimes, des accidents de la circulation, ou encore des accidents du travail) illustre parfaitement la démarche préconisée à travers le modèle de l'ingénierie psychosociale. En préalable, plusieurs études ont permis de dresser un bilan (étape 1) des procédures mises en œuvre par les professionnels concernés, et même de comprendre la théorie que les policiers se font du témoin. En effet, les policiers sont convaincus que les témoins possèdent de faibles performances mnésiques et produisent beaucoup d'erreurs. Ils vont donc les « aider » en leur imposant une succession de questions spécifiques planifiées en fonction des nécessités du procès-verbal; ce faisant ils influencent le témoin et n'exploitent pas sa mémoire. Plusieurs propositions ont été dégagées de ce bilan (étape 2 : accorder une large place au rappel libre des faits, éviter le recours systématique à des questions, respecter le cheminement mental du témoin, etc.) et de la littérature scientifique sur la mémoire humaine 
(principe d'une remise en contexte mentale préalable au témoignage, utilisation des effets de récence, variation des plans de récupération, désactivation des schémas de narration). Ces différentes propositions ont été testées dans un cadre d'application de manière à constituer une technique opérationnelle (étape 3). L'efficacité de cette technique en comparaison de la procédure habituelle a été mesurée à de nombreuses reprises en laboratoire et sur le terrain au moyen d'expérimentations comme de recherches-action. On obtient ainsi, en moyenne, 30 \% d'informations exactes supplémentaires avec la technique de l'entretien cognitif par rapport à l'entretien standard de police, sans accroissement du nombre d'erreurs ou du nombre d'informations inventées par le témoin. Ce modèle d'action ayant été validé, un vaste plan de formations des officiers de police a été mis en place (étape 4), le dispositif de formation étant régulièrement optimisé grâce à une démarche systématique d'évaluation de l'efficacité des apprentissages.

\section{Propos SUR LA MÉTHOdE ET SUR LA DÉONTOLOGIE}

À ce point il nous apparaît nécessaire de questionner quelque peu les méthodes de recherche en psychologie sociale, en particulier quand on parle de ses applications et de son applicabilité. Tout chercheur peut avoir somme toute plusieurs objectifs : (1) décrire les événements et s'assurer de moyens fiables pour les mesurer, (2) découvrir les covariations ou associations de phénomènes, (3) découvrir les relations causales, c'est-à-dire les lois de cause à effet qui unissent les phénomènes. Pour simplifier le propos, les méthodes corrélationnelles permettent de mettre en évidence des liens entre différentes variables isolées sur le terrain, mais que l'on ne peut pas forcément manipuler (par exemple entre un mode managérial de chefs de service et le degré de stress perçu des collaborateurs). La méthode expérimentale pour sa part vise à mettre en évidence des relations de cause à effet. Or les termes « application » et « applicabilité » laissent penser que les seules méthodes utilisables relèvent de méthodes descriptives et corrélationnelles (observation systématique, corrélations, études génétiques, études « ex post facto»), mais relèvent moins - voire pas - de l'expérimentation. Nous nous inscrivons en faux contre cette idée puisque si l'expérimentation est évidemment une réduction de la réalité (dans le sens d'une miniaturisation, de par le fait que le phénomène observé est abordé via la manipulation d'une à trois variables choisies parmi l'ensemble de variables potentiellement en jeu), la seule manière de démontrer une relation causale entre des 
variables est de créer soi-même la cause et de montrer que, ce faisant, on obtient l'effet annoncé dans la relation causale. Ainsi dans l'optique expérimentale, le chercheur crée les conditions dans lesquelles les sujets sont placés, puis il en observe sur eux leur effet. Toutefois le fait d'avoir « créé » les conditions de l'observation n'implique pas que cette dernière soit artificielle. Par exemple, à partir d'une question de terrain relative à l'usage du « 360 degrés $^{4}$ », Matuchet, Somat et Testé (2005) menèrent une première recherche expérimentale de laboratoire consistant en une simulation d'évaluation 360 degrés auprès d'étudiants en fin de cursus d'étude de commerce. Cette simulation consistait à vérifier la possibilité d'adjoindre une consigne métaperceptive (dont le projet consistait à demander à chaque évaluateur la perception qu'il pouvait avoir de l'évaluation dont le manager était l'objet) et une mesure de la performance basée sur des traits et des comportements d'efficacité professionnelle et d'attractivité professionnelle. L'évaluation $360^{\circ}$ est apparue indépendante de l'attribution d'efficacité managériale. Une seconde recherche expérimentale de terrain a consisté cette fois à proposer une telle évaluation en milieu industriel. Les résultats ont montré que le manager qui bénéficiait de perceptions favorables était jugé plus efficace. Cet ensemble de recherches a permis de conclure que l'évaluation $360^{\circ}$ n'avait pas l'intérêt qu'on lui suppose pour prédire la performance organisationnelle, mais que cet outil pouvait trouver sa légitimité dans sa capacité à optimiser le management participatif. Il donne, de fait, par l'évaluation du supérieur hiérarchique, à chaque participant, la possibilité de construire ensemble les modalités du management de l'équipe. Comme dans le cas des recherches précitées sur l'entretien cognitif, on voit bien ici ce qu'une recherche expérimentale peut apporter à la compréhension d'un phénomène psychosocial et les leviers qu'elle peut apporter pour l'action. Une autre remarque relative à l'expérimentation s'impose : si les variables retenues puis manipulées ont effectivement l'effet prédit, un levier pour l'action devient évidemment disponible. Si l'on veut construire, peut-être vaut-il mieux avoir les bons outils. Enfin, manipuler des variables suppose le respect d'une certaine déontologie. Il en découle un principe essentiel : la

4. Le $360^{\circ}$, ou «évaluation à évaluateurs multiples», ou encore "feedback à sources multiples », est un processus évaluatif qui a pour objectif de fournir un retour d'information sur la performance de responsables de différents niveaux hiérarchiques. Cette appellation trigonométrique tient dans le fait que le manager, par le biais d'un questionnaire, peut comparer la perception de son management à celle de son entourage professionnel : ses proches collaborateurs, ses collègues, son supérieur hiérarchique et parfois quelques clients internes ou externes à l'entreprise. 
liberté de refuser de participer à une expérience. Quand ils acceptent, les sujets doivent nous quitter (1) avec une image d'eux-mêmes non dépréciée par rapport à celle qu'ils avaient en arrivant, (2) sans avoir acquis de raisons de renoncer à leurs projets personnels, (3) ni plus ni moins bons ou mauvais qu'ils le seraient s'ils ne nous avaient pas connus (voir par exemple Beauvois, Roulin, \& Tiberghien, 1990).

\section{Au FIL DES CHAPITRES...}

Nous verrons dans cet ouvrage que la psychologie sociale, en tant que branche de la psychologie scientifique, a une véritable utilité puisqu'elle permet l'obtention du changement social en particulier en modifiant les pratiques professionnelles. Nous verrons que ces modifications de pratiques peuvent s'envisager dans divers domaines. Les champs de recherche présentés ici ne couvrent pas, loin s'en faut, la totalité des applications actuelles et potentielles de la psychologie sociale (un tel objectif dépasse largement le cadre de cet ouvrage). Nous avons choisi de présenter un florilège de recherches qui nous ont semblé particulièrement illustratives ${ }^{5}$, laissant ensuite le soin au lecteur de continuer son cheminement dans la démarche initiée par l'ouvrage.

Nous tendant un miroir, les chapitres d'Ewa Drozda-Senkowska et de Dominique Oberlé et Benoît Testé ont une dimension clairement réflexive et montrent aussi l'insertion socio-historique des préoccupations des chercheurs. Dans son prologue, Ewa Drozda-Senkowska propose une réflexion épistémologique sur la démarche scientifique et ses finalités. Elle montre clairement que la pensée des philosophes et des chercheurs effectue au fil de l'histoire un mouvement de balancier entre deux polarités qui ne sont pas aussi opposées qu'on pourrait le penser de prime abord. Elle s'interroge sur trois questions qui sont aussi des confusions fréquentes: la différence entre la recherche appliquée et la recherche dite " pure » ou «fondamentale »; la confusion entre la « recherche de terrain» et la " recherche appliquée "; et la confusion entre la « recherche appliquée » et l'« applicabilité de la recherche ». Elle montre aussi que la méthode, parce qu'elle est commune à toute science, peut réunir ce qui peut sembler initialement différent ou épars. Enfin elle insiste sur la nécessité de la diffusion de la recherche à l'extérieur des cercles scientifiques, ce qui est d'ailleurs la fonction première de cet ouvrage. Dominique Oberlé et

5. Dans chaque chapitre, les encadrés font référence à quelques-unes des communications affichées lors du colloque de l'ADRIPS. 
Benoît Testé présentent la manière dont les chercheurs, au fil de l'histoire et des nécessités économiques et politiques, se sont emparés de la notion de "groupe ", un des concepts fondamentaux des sciences humaines et sociales. Ils montrent ainsi comment le comportement de l'individu au sein d'un groupe a été appréhendé. Analysant ensuite un corpus important de publications, ils montrent comment est conçu le caractère « appliqué » des recherches en psychologie sociale, en particulier les recherches portant sur le groupe. Par exemple, est-il considéré comme un objet de recherche ou comme un moyen d'action? S'intéresse-t-on à des problèmes sociaux généraux s'exprimant dans des contextes variés, ou à des problèmes spécifiques se posant forcément dans des contextes particuliers? De cette analyse découlent des critères permettant d'appréhender l'applicabilité de la recherche. Sont proposées enfin quelques pistes de réflexion concernant ce qui peut être attendu de la recherche appliquée en psychologie sociale et à quelles conditions elle peut être mise en œuvre, au regard en particulier du rôle du psychologue social.

Les deux chapitres suivants ont trait à l'influence, et plus particulièrement aux déterminants des comportements. Julien Cestac et Thierry Meyer traitent d'une question fondamentale : peut-on prédire le comportement des gens à partir de ce qu'ils racontent? En 1934, La Piere en avait déjà pointé les limites. Pourtant aujourd'hui encore, si l'on en croit l'observation quotidienne tout au moins, les personnes paraissent croire en l'existence d'une congruence des attitudes et des comportements ${ }^{6}$. Outre une revue de la littérature sur la question des attitudes, Cestac et Meyer présentent ici le Modèle du Comportement Planifié (MCP) d'Ajzen. Une règle de base est que si l'on souhaite prédire un comportement spécifique, il faut alors interroger les croyances, attitudes et intentions à l'égard de ce comportement spécifique, dans un espace-temps bien délimité. Le MCP postule que les personnes ont d'autant plus de chances de s'engager dans un comportement qu'ils en ont l'intention, qu'ils évaluent favorablement ce comportement, qu'ils croient que les autres le jugent favorablement, et qu'ils estiment le maîtriser; et les auteurs proposent d'autres prédicteurs pour élargir le modèle, qui a déjà été mis en œuvre dans de très nombreux domaines comme l'éducation, la santé, les transports, l'environnement, ou encore les choix politiques. Il est d'autres manières de traiter des déterminants du comportement. Robert-Vincent Joule et Nicolas Guéguen présentent des stratégies pouvant être mises en

6. Ce point indique clairement à lui seul la nécessité de la diffusion de la recherche prônée par Ewa Drozda-Senkowska. 
œuvre pour emporter le changement comportemental, issues des théories de l'engagement - défini comme le lien qui unit la personne à son acte : plus je peux dire que mon comportement me correspond (ou correspond à mes valeurs, à la définition que j'ai de moi, à ma nature...), plus je suis engagé. À la différence des pressions explicites d'une autorité par exemple, l'engagement développe un " cercle vertueux » qui en quelque sorte s'auto-alimente. Les auteurs présentent les recherches ayant fondé le champ, mais aussi des études plus récentes, dont les implications sont évidentes : conduites altruistes (aider ou prendre soin de la santé de son enfant), se préserver (fumer moins, adopter des comportements de sécurité au travail) ou encore produire des comportements écologiques. De récents développements prennent en compte les déterminants langagiers de l'engagement que les auteurs appellent la « communication engageante ». Quelques implications, entre autres au niveau des comportements écologiques, sont présentées. Évidemment la question de l'éthique (notamment sur l'usage qui peut être fait des techniques d'engagement) est sous-jacente à l'ensemble de ces recherches. Elle était d'ailleurs déjà évoquée par Joule et Beauvois dès 1987.

Certains comportements humains sont problématiques, c'est le cas de l'agression. Si le comportement agressif a parfois été vu comme naturel (en particulier parce qu'en partie lié à des mécanismes hormonaux), le chapitre de Laurent Bègue en présente les mécanismes psychosociaux, via le modèle général de l'agression. Sans occulter les facteurs internes pouvant amener une personne à l'acte agressif, l'auteur décrit l'ensemble des facteurs en jeu dans les situations d'agression. On y voit clairement que l'agression est aussi apprise et dépend de facteurs psychosociaux, comme les normes groupales et l'affiliation au groupe, ou encore des variables professionnelles. L'auteur montre la nécessité de prendre également en compte la manière dont la personne perçoit, ressent, et interprète la situation (entre autres, ses propres réactions). Finalement l'agression est très dépendante des contextes; mieux les connaître devrait permettre d'agir avec plus de justesse. L'auteur présente aussi la méthodologie riche et variée permettant au chercheur d'approcher et de mieux comprendre le comportement agressif, allant de l'observation à l'expérimentation en passant par les enquêtes d'auto-déclaration ou de victimation.

Chacun peut, théoriquement, être un jour désigné comme juré par la Justice. Porteur de ce rôle imposé par l'autorité judiciaire, il faudra décider de la culpabilité ou de l'innocence d'un prévenu. Candy Sabatier et Georges Schadron apportent des réponses à la question : comment allonsnous prendre cette décision, dans ce contexte si particulier? Si certains 
déterminants des décisions des jurés peuvent paraître, en première analyse, plutôt évidents (les croyances, les attentes, les protagonistes...), d'autres le sont moins, ce qui pose la question des déterminants non conscients des jugements. On y voit que ces derniers relèvent d'automatismes permettant une adaptation optimale à l'environnement, mais pouvant avoir des effets indésirés/indésirables. Par exemple, le fait de se savoir connu par autrui (ce qui est le cas des jurés), d'être jugeable, amène les personnes à être plus influençables, plus « déterminables ». Les recherches présentées ici montrent comment leurs jugements peuvent être affectés dans cette situation, entre autres par les attentes normatives implicites.

Sous un autre angle, essentiellement cognitif, Gaëlle Villejoubert et Valérie Le Floch traitent également des jugements et des décisions. Elles en évoquent les biais, les écueils et les limites. Par exemple, elles montrent que les prévisions subjectives sont quasiment toujours plus erronées que celles d'une simple analyse statistique. Il apparaît ainsi que l'homme n'agit pas forcément comme un statisticien naif, que nos décisions ne sont pas particulièrement rationnelles, et sont fréquemment biaisées, entre autres parce que l'on se focalise sur l'information individualisante. Des exemples concrets de tels biais, relatifs aux situations médicales ou de recrutement, sont présentés. Les auteures proposent enfin une méthodologie d'aide au jugement et à la décision, pouvant aider les professionnels à émettre des jugements plus justes et à prendre des décisions plus adaptées.

Qu'est ce que la santé? Qu'est-ce que la psychologie de la santé? Qu'est-ce qu'un comportement de santé? Quels leviers utiliser pour la prévention? Florence Terrade examine les apports de la psychologie à la prévention dans les comportements relatifs à la santé. Différents courants de recherche, et les méthodologies utilisées, sont ici présentés. Il apparaît, comme évoqué par Cestac et Meyer, que connaître les intentions des gens n'est pas suffisant pour prédire leurs comportements de prévention. Ici encore, il est nécessaire de prendre en compte les croyances et les connaissances initiales, mais aussi de considérer les différents niveaux de prévention, non pas indépendamment les uns des autres, mais dans leur globalité. Enfin, à l'instar des recherches sur l'agression, les méthodologies utilisées sont variées, et contribuent probablement à une meilleure compréhension des comportements.

Comment une personne explique-t-elle les événements? Cela joue-t-il un rôle dans sa réussite ou ses échecs? Pascal Pansu et Philippe Sarrazin exposent les applications issues des travaux sur les théories de l'attribution. Ces applications ils les trouvent dans le domaine de l'éducation et 
plus particulièrement dans le champ des études universitaires. Comme chez Sabatier et Schadron au niveau des jugements, on voit apparaître un fonctionnement largement automatique des attributions. Les auteurs convoquent ici quatre modèles susceptibles d'expliquer la réussite académique. Ils présentent et discutent ensuite une série de recherches de reconversion attributionnelle (amener les élèves à expliquer autrement leurs échecs), et dont la fonction est d'améliorer leur réussite aux examens. Si de nombreuses questions persistent, la reconversion attributionnelle apparaît bien à la fois comme un objet de recherche fertile et comme un potentiel outil d'amélioration.

Le psychologue dispose également de stratégies d'action pour comprendre les processus psychosociaux impliqués dans le recrutement et favoriser l'insertion professionnelle. Steiner, Roques, Pichot et Maisonneuve font le tour de différents champs de la psychologie sociale impliqués dans les situations professionnelles. Si certaines théories relèvent davantage d'une préoccupation concernant des problèmes sociaux généraux s'exprimant dans des contextes variés, comme le disaient Oberlé et Testé, d'autres réfèrent essentiellement à des préoccupations spécifiques. Dans le premier cas, les processus de catégorisation permettent de mieux comprendre le jugement envers les personnes recrutées, dans le second l'attachement ou la justice organisationnelle permettent de mieux décrire voire de mieux comprendre les comportements en entreprise. Quoi qu'il en soit, les auteurs exposent sans ambigüité quelques théories qui incontestablement participent à l'action. Ils montrent également que les problématiques issues du terrain peuvent dans certains cas alimenter la réflexion du chercheur.

Gérard Guingouain et Jean-Luc Hannequin s'interrogent enfin sur l'articulation du monde de la recherche et du monde économique. Ancrant leurs réflexions sur celle de Drozda-Senkowska, ils développent la question de la valorisation de la recherche, en pointant les différents freins rencontrés, mais aussi en proposant des ouvertures, allant du décloisonnement disciplinaire au développement de synergies. Des exemples concrets de collaborations illustrent d'ailleurs leur réflexion. On retiendra que pour eux, la distinction « recherche fondamentale - recherche appliquée » est peu opérante quand on parle de valorisation, et qu'en définitive c'est la robustesse des cadres théorique et méthodologique qui incite les acteurs du monde économique à faire appel aux compétences des chercheurs en psychologie sociale.

Pour conclure, on voit nettement émerger dans cet ouvrage une réelle unité dans la diversité des champs présentés. D’abord, une unité 
théorique : d'une part, dans le fait que le comportement des personnes est toujours relié à la manière dont elles perçoivent la situation et au contexte social; d'autre part, dans la convocation des mêmes modèles dans différents domaines. On voit ainsi clairement apparaître la relative indépendance de la théorie par rapport au champ d'application. Ensuite, une unité méthodologique : la démarche, les outils sont indépendants du champ et de la finalité de la recherche. Enfin, dans chaque champ présenté ici (mais la réflexion vaut évidemment pour tous les autres), les chercheurs se préoccupent certes de questions d'application ou d'applicabilité de leurs travaux, mais on a bien perçu qu'ils sont également guidés par des préoccupations éthiques, formulées parfois très explicitement. Cet intérêt peut d'ailleurs être à l'origine de leur démarche comme il peut en résulter. Qu'elles soient premières ou secondes, ces préoccupations éthiques ont été évoquées de différentes manières dans cet ouvrage. Souhaitons que le lecteur garde à l'esprit l'idée de Cestac et Meyer (dans cet ouvrage) : « Le chercheur n'est ni un missionnaire, qui ne sert que des causes au service de sa communauté, ni un mercenaire prêt à servir toutes les causes. »

\section{Références}

Beauvois J.-L. \& Ghiglione R. (1989), « Psychologie sociale et organisations », in J.-M. Monteil \& M. Fayol (dir.), La psychologie scientifique et ses applications, Grenoble, Presses universitaires de Grenoble, p. 307-322.

Beauvois J.-L., Roulin J.-L. \& Tiberghien G. (1990), Manuel d'études pratiques de psychologie, Paris, PUF, coll. « Le Psychologue ».

Demarchi S. \& Py J. (2007), «L'entretien cognitif : son efficacité, son application et ses spécificités », Revue québécoise de psychologie.

Fleishman E. A. (1979), « The new applied psychology: An international perspective », International Review of Applied Psychology, 28, p. 61-14.

Fleishman E. A. (1999), « Applied psychology: An international journey », American psychologist, 54, p. 1008-1016.

FÉReC N., PANSU P., Py J. \& SOMAT A. (en révision), « Une approche socio-normative de l'accompagnement des demandeurs d'emploi : un module de formation à la présentation de soi en situation d'évaluation », Orientation scolaire et professionnelle.

Guingouain G. \& Le Poultier F. (1994), À quoi sert aujourd'hui la psychologie sociale?, Rennes, Presses universitaires de Rennes.

Jacques B., Somat A., Aldrovandi M., Gingouain G. \& Rault L. (2002), "L'intériorisation des valeurs de l'entreprise comme enjeu du management participatif », Cahiers internationaux de psychologie sociale, 56, p. 32-46.

Joule R.-V. \& Beauvois J.-L. (1987, rééd. 2002), Petit traité de manipulation à l'usage des honnêtes gens, Grenoble, Presses universitaires de Grenoble.

Kergreis S., Somat A. \& Testé B. (2003), « Les valeurs engagées dans les discussions de groupe : Trois études dans le cadre de concertations agri-environnementales », Actes du colloque "Normes sociales et processus cognitifs ", Poitiers. 
Kergreis S., Somat A. \& Testé B. (2009), « La concertation conduit-elle à changer les points de vue? Une étude psychosociale sur les bords de champs en Bretagne », in L. Mermet \& M. Berlan-Darqué (dir.), Environnement : Décider autrement. Nouvelles pratiques et nouveaux enjeux de la concertation, Paris, L'Harmattan.

La Piere R. T. (1934), «Attitudes vs action », Social forces, 13, p. 230-237.

Matuchet S., Somat A., Testé B. \& Lucet E. (2005), « L'émergence et le développement de l'évaluation 360 degrés, vecteur du management participatif », Psychologie du travail et des organisations, 4.

Py J., Somat A. \& Baillé J. (1998), Psychologie sociale et formation professionnelle, Rennes, Presses universitaires de Rennes.

Py J. \& Somat A. (2007), « Ingénierie psychosociale : un modèle de recherche appliquée et d'intervention », in A. Trognon \& M. Bromberg (dir.), Psychologie sociale et ressources humaines, Paris, Presses universitaires de France, p. 161-176.

PANSU P., Py J. \& SOMAT A. (à venir), L’ingénierie psychosociale et cognitive : état des lieux et méthode pour l'action.

Rosenzweig M. R. (dir.) (1992), International psychological science: Progress, problems, and prospects, Washington DC, American Psychological Association.

Rosenzweig M. R. (1999), "Community and change in the development of psychology around the world », American Psychologist, 54, p. 252-259.

Wilpert B. (1998, August), Presidential address, Address presented at the 24th International Congress of Applied Psychology, San Francisco, CA. 